\title{
ENCUADRE DE UN MODELO ALTERNATIVO PARA EL ESTUDIO DE LA CALIDAD UNIVERSITARIA. UNA PROPUESTA PARA PSICOLOGÍA
}

\author{
Oswaldo Orellana Manrique*, Daphne Orellana García**
}

\begin{abstract}
RESUMEN
La presente es una investigación exploratoria sobre la calidad universitaria. Realiza una revisión histórica sobre las funciones de la universidad y una revisión contemporánea acerca del concepto de calidad universitaria, debatiendo su contenido y extensión, principalmente en su aplicación en el Perú. Aborda los modelos introducidos en América Latina y sus manifestaciones en la formación de psicólogos, centrándose en la Universidad Nacional Mayor de San Marcos y concluyendo con la propuesta de tópicos para evaluar la calidad a partir de un modelo para la autoevaluación.
\end{abstract}

Palabras claves: Universidad, calidad universitaria, acreditación, autoevaluación, currículo universitario.

\begin{abstract}
Present is an exploratory investigation on the university quality. It realizes a historical review on the functions of the university and a contemporary review brings over of the concept of university quality, Debating his content and extension, principally in his application in the Peru. It approaches the models got in Latin America and his manifestations in psychologis ts' formation, Centring on the National Major University of San Marcos And concluding with the offer of topics to evaluate the quality from a model for the autoevaluation.
\end{abstract}

Keywords: University, university quality, crediting, autoevaluation, university curriculum.

\footnotetext{
Docente Principal Investigador del IIPs. (UNMSM) (E-maill: ovaorellana@yahoo.es)

"Psicóloga, Investigadora colaboradora.
} 


\section{INTRODUCCIÓN}

El presente trabajo de investigación se planteó los siguientes objetivos: (1) Realizar una revisión teórica de los principales aspectos de referencia, difundidos en nuestro país acerca de la calidad universitaria, (2) Identificar las tendencias conceptuales que se emplean para abordar la calidad universitaria, (3) Promover el debate sobre la calidad universitaria en la formación de psicólogos, (4) Plantear alternativas para llevar adelante investigaciones integrales sobre la calidad en la formación de psicólogos, (5) Establecer líneas prioritarias para investigaciones pertinentes.

Partimos de la premisa que históricamente la institución encargada de validar las distintas expresiones del conocimiento, principalmente el científico, ha sido la universidad, pues ésta se constituía como un espacio cultural donde los ciudadanos acudían a buscar la verdad, en base a las indagaciones realizada por estudiosos de la filosofía, la ciencia, la literatura y otras formas de conocimiento que enriquecían el saber de la humanidad. Los ciudadanos, principalmente jóvenes eran partícipes de un debate alturado y obtenían un reconocimiento especializado en determinadas practicas del conocimiento que ejercían en su comunidad. Con el transcurrir del tiempo estas funciones típicas fueron adquiriendo formas culturales especificas, como son la producción del conocimiento (investigación), el otorgamiento de grados académicos y los permisos certificados para ejercer una practica especializada en la sociedad, que se conocen como licencias profesionales. De esta manera podemos concluir que la esencia de la universidad es la investigación y la producción de conocimientos, y sus funciones derivadas son el otorgamiento de grados académicos y títulos profesionales.

Transcurridos mil años del funcionamiento de la universidad y después de haber pasado épocas de luces como son el renacimiento y la ilustración en el antiguo mundo, y el apoyo a la lucha anticolonial en América, ingresamos a una nueva época de dimensión mundial con un carácter económico-financiero de apertura y dominación de mercados, bautizado con el nombre de globalizacion, que en realidad es una fase del capitalismo, caracterizada por nuevas formas de dominación y hegemonía del gran capital financiero y comercial.

Estructuralmente la humanidad se encuentra en un cambio de época, donde el orden social y cultural vienen adecuándose a las nuevas circunstancias de ruptura estructural. Sin embargo es necesario hacer notar la relativa autonomía que las instituciones culturales, como la universidad, han tenido en ocasiones similares, el de no caer fácilmente en la sumisión de los sistemas de dominación y en cambio buscar desarrollar salidas alternativas, en defensa del pensamiento innovador y/o el ejercicio del pensamiento divergente, comprometido con los ideales supremos de la humanidad y el bienestar de la población.

Es en este contexto que aparece la predica de la calidad, como exigencia para la sobrevivencia institucional de los centros educativos de todos los niveles, incluida la universidad. La calidad como eje de eficiencia institucional proviene de las empresas de producción y las empresas de servicio, las cuales consiguen un certificado técnico de buen funcionamiento, categorizado como acreditación de la calidad.

Así se introduce una nueva terminología estandarizada en las empresas privadas extendidas al ámbito educativo, como son las siguientes: gerencia, planeamiento estratégico, competitividad, satisfacción del cliente, mejora continua, productividad o rendimiento de cuentas, liderazgo y eficiencia. 
De otro lado se difunden modelos que deben de aplicarse en la planificación de la calidad.

Haciendo una revisión de referencias acerca de la calidad hemos encontrado dos, muy difundidos en el ámbito educativo, el modelo sistemico y el modelo de gestión, adaptados a la educación, y por lo tanto, unilaterales, en tanto enfocan la educación como un servicio, similar a un negocio de cualquier naturaleza, sosteniendo que la "satisfacción del cliente" encausa el criterio matriz, en base al cual se proyecta la mejora continua.

Ambos modelos en la planificación aportan el control de una estructura funcional, ordenada y "disciplinada", como efectos inmediatos de la evaluación. En el primer nivel referida a la planificación estratégica y en el siguiente los análisis comparativos de la institución con otras similares a través de la metodología de la selección de pares.

En el Perú esta cultura de calidad se introdujo en la ultima década del siglo anterior, con una prédica ideológica que la educación privada tiene calidad y la educación publica no la tiene. Para tal efecto tuvo como referencias un conjunto de indicadores "objetivos" que muy pocos, por no decir nadie se atrevía a cuestionar. Se identificaron "fuertes" deficiencias tecnológicas y de actualización profesional, y el resultado negativo de evaluaciones internacionales sobre el rendimiento académico de los escolares. A nivel universitario se manipuló el deterioro de la infraestructura, ya sea por falta de mantenimiento y la negativa de los gobiernos para invertir en equipamiento y edificaciones, así como aumentar las remuneraciones a los docentes; y el aspecto ideológico de la politización de sus miembros, docentes, estudiantes y trabajadores, que exigen mejor trato.

Heinz Dieterich (1997), analista internacional de la educación, compañero de pensamiento de Noam Chomsky, sostiene lo siguiente "es ciertamente demagógico sostener que la miseria latinoamericana sea el resultado de la deficiente educación del subcontinente, cuando hay una serie de variables determinantes de igual o mayor importancia como son la deuda externa, la corrupción de las elites, el proteccionismo del primer mundo que le brinda diez veces más de la que da en términos de "ayuda"; las condiciones del mercado, la falta de ahorro interno, la fuga de capitales. La pobreza absoluta y la distribución extremadamente desigual del ingreso, los gastos militares, el impedimento de cualquier proceso de reforma profunda en pos de intereses populares -como en Nicaragua o Cuba-por parte de Estados Unidos y sus aliados; el desinterés y la ineptitud de la clase empresarial criolla para la innovación científica y tecnológica..."1

Dos sujetos son desconocidos en el modelo de calidad: La docencia y el estudiante, remplazados por la gestión de indicadores estadísticos y la atención al cliente, absurdo desde todo punto de vista, nada justifica que al estudiante se le llame cliente, e inclusive se "avanza" y se dice que hay clientes internos y clientes externos. De otro lado es impertinente afirmar que los profesores son los proveedores de conocimientos, en la misma lógica del suministro de insumos.

La certificación de la calidad es orientada por estándares internacionales formulado y sistematizados por la ISO o International Standars Origanization (Organización Internacional de Normalización). Históricamente se asoció ISO con el vocablo griego ISOS, que significa igual, y fue fundada en el año 1946 en Ginebra por más de 100 países, que se plantearon homogenizar sus servicios y productos de acuerdo a la competitividad de proveedores y exigencias de sus clientes. Con el transcurrir del tiempo y habiendo logrado un nivel mínimo de coordinación para la vigencia de servicios y productos, se editaron un conjunto 
de normas que se les conoce como familias de normas ISO 9000, relativas principalmente a los sistemas de gestión y la terminología de la gestión de calidad. Posteriormente aparece las normas ISO 9001 y la ISO 9004. ISO 9001-2000 La primera fija los requisitos para los sistemas de gestión de la calidad que responda a las exigencias de sus clientes, y la versión ISO 9004 enfatiza en los sistemas de eficiencia y eficacia de la gestión de calidad y la ISO 9001-2000 se basa en el enfoque basado en procesos (Planificar, Hacer, Verificar y Actuar).

La gestión de calidad referidas a las ISOs es el buen desempeño de la gestión dentro de una organización, sistematizadas en ocho principios, que nominalmente son los siguientes: (1) Organización orientada al cliente, (2) Liderazgo, (3) Participación del personal, (4) Enfoque basado en procesos, (5) Enfoque del sistema hacia la gestión, (6) La mejora continua, (7) Toma de decisiones basadas en los hechos y, (8)Relaciones mutuamente beneficiosas con los proveedores.

Como se puede apreciar estos instrumentos para evaluar la calidad están muy lejos para su aplicación en el campo educativo, ya sea de la educación básica o de las superior universitaria, en el mejor de los casos, y con muchas limitaciones se podría aplicar a un aspecto del funcionamiento, específicamente al área administrativa.

La calidad universitaria necesariamente tiene que pasar por el temas de las reinvindicaciones, similar a lo que se plantea el desarrollo humano en el objetivo de lograr la calidad de vida de la población. Así tenemos que en un contexto de salida de un conflicto armado interno, en el Perú la Comisión de la Verdad y Reconciliación Nacional, recomienda hacer reparaciones a las víctimas, del mismo modo en la universidad tendríamos que señalar que el objetivo de lograr la calidad se inicia con una mayor atención presupuestaria e interés por la investigación, así como el cumplimiento de la homologación docentes con los magistrados, establecida en el art. 53 de la ley universitaria vigente desde el año 1983.

En tal sentido nuestro problema de Investigación quedó planteado en los siguientes términos ¿Qué referencia conceptual es la más adecuada para abordar la calidad universitaria?, ¿Qué aspectos relevantes hay que tener en cuenta para iniciar una evaluación de la calidad universitaria en la formación de psicólogos?

Ortega y Gasset pensador del siglo pasado quien ejerció gran influencia en muchas generaciones universitarias escribió un ensayo muy difundido en los ambientes juveniles titulado "Misión de la Universidad"2, aquí propone tres principios universitarios: a) la transmisión de la cultura, b) la enseñanza de profesionales intelectuales, y c) la investigación científica y formación de futuros investigadores.

La universidad no se limita a formar profesionales, sino es necesario que estos tengan y sean promotores de la cultura, específicamente de la cultura nacional que favorezca la identidad, y el otro aspecto del mismo proceso es la formación de investigadores, comprometidos y capaces de entender los problemas de su disciplina articulados con otros conocimientos, que es la fuente de la interdisciplinariedad.

En el mismo sentido los expertos de la educación universitaria lo expresan en distintos terminos, como podemos apreciar en el siguiente pensamiento. "En los países del primer mundo la educación superior se expande y diversifica. No está hoy sujeta a un formato u objetivo educativo único. $\mathrm{Al}$ absorber nuevos públicos, ella pasa a cumplir nuevas funciones y a diferenciarse internamente, sea esta diferenciación intencional o no. Crece, además, el 
contingente de adultos interesados en ampliar su formación básica general o en calificarse para un mercado profesional más exigente. Se amplían las formas de educación continua, que posibilitan que la población adulta retorne una o más veces al sistema de enseñanza a lo largo de su vida. Se expande, de forma aún más avasalladora, el peso de la actividad de investigación y de producción científica en el interior de las grandes universidades, con repercusiones importantes sobre las demás funciones de esas instituciones" ${ }^{3}$.

En San Marcos las autoridades universitarias encargadas de la administración desde el año 2001 , vienen promoviendo un modelo de universidad denominada "Universidad abierta al futuro", planteándose tres grandes líneas estratégicas": a). Promoción de la calidad académica, b). Financiamiento público y sostenibilidad, y c). Gobierno electrónico: Una gestión moderna y eficiente. Sobre la calidad académica afirman lo siguiente: "La promoción de la calidad académica es una de las más grandes tareas asumidas por todas las universidades públicas en el Perú y América Latina. Las tres últimas décadas del siglo XX trajeron consigo masificación de estudiantes, reducción del apoyo estatal, deterioro de la condición del docente y finalmente pérdida de la calidad. En otros países como en México, Brasil o Chile, han afrontado estos problemas renovando sus legislaciones universitarias y asignando mayores recursos públicos a la universidad. En el Perú, durante este mismo periodo, la violencia social, la crisis política y una postración económica sin precedentes, han impedido tomar medidas similares y más bien han derivado en una constante y compleja gobernabilidad. Mejorar la calidad en San Marcos no es responsabilidad exclusiva de la administración central, sino más bien una tarea que demanda la participación de todos, y que además es necesaria, urgente e ineludible y por eso no importa que marchemos contra la corriente, pero indudablemente rumbo al futuro" ${ }^{5}$, finaliza un documento del movimiento Acuerdo Institucional Sanmarquino, al que pertenecen las autoridades universitarias de la alta dirección.

Los objetivos estratégicos de la Universidad Nacional Mayor de San Marcos se encuentran plasmados en el Plan Estratégico Institucional (PEI) 2002-2006 6 : 1. Formar profesionales integrales: Competitivos, cultos. Con espíritu crítico y creativo, líderes en su especialidad, generadores de conocimientos, con valores y comprometidos con el desarrollo de la sociedad, Docencia 2. Contar con una plana docente de alto nivel académico y pedagógico. Con compromiso ético, moral y social, que contribuya a la formación de profesionales integrales, producción de conocimientos, 3. Establecer la generación de conocimientos, con énfasis en la investigación científica, tecnológica y humanística, como eje fundamental del desarrollo de la universidad, orientado a resolver los problemas prioritarios de la sociedad, Integración social, 4. Hacer de la integración social un pilar para el desarrollo de la universidad, Estado, empresas e instituciones sociales, hacia un desarrollo integral y sostenible, Gestión 5. Desarrollar una cultura organizacional de excelencia basada en principios y valores que permitan una gestión de alta calidad.

En nuestro medio ha tenido relativa difusión la enseñanza basada en competencias, para la cual la formación universitaria debe ser un periodo de adiestramiento laboral del futuro profesional, debiéndose de producir "programas de adiestramiento, basado en el análisis ocupacional. La educación universitaria se reduciría a un programa de operaciones, desglosados en programas instruccionales.

El modelo ha sido tomado de las empresas norteamericanas que mantienen un programa de adiestramiento laboral, que incluyen los materiales y las operaciones requeridas. 
El programa de adiestramiento tiene las siguientes fases: Análisis, diseño, desarrollo, implantación y evaluación El diseño corresponde a la preparación del curriculum global, es decir lo que corresponde al aprendizaje, luego viene la enseñanza o el adiestramiento en los distintos contextos, en el salón de clase, laboratorio, en tercer lugar se plantean los objetivos de aprendizaje donde debe fusionarse la operación y el comportamiento llamado el objetivo final de ejecución (OFE).

En este enfoque la universidad tiene que subordinarse al análisis del trabajo y de las operaciones (ATT) que se está llevando a cabo en el mundo del trabajo profesional. El desarrollo o tercera fase se refiere al cómo, o procedimiento y habilidades debe de aplicarse. La implantación se refiere a los recursos humanos disponibles y los recursos materiales requeridos, la evaluación se realiza en base a los objetivos.

Sin embargo es necesario señalar grandes deficiencias en la planificación social, como las señala Verussi de la siguiente manera "Cualquier intento de describir y analizar la dinámica cambiante de las relaciones entre educación superior y empleo en América Latina, enfrenta el problema de la diversidad de sistemas de empleo y de educación superior así como el de la falta de información pertinente disponible ${ }^{7 "}$.

Los criterios empleados para la enseñanza basada en competencias son los siguientes: La importancia de la operación, la dificultad del aprendizaje (o la dificultad de realizar operación) y la frecuencia con que se realiza.

Volvemos a precisar que el eje de la universidad es la producción de conocimientos, entiéndase este proceso como resultante de la investigación. Esta es la orientación internacional para la acreditación, así lo muestran los resultados del ranking universitario mundial realizado por la universidad de Shanghai Jiao Tong de la República Popular China (2003). Los criterios empleados para realizar dicho ranking, resumidos por Carlos García Bedoya, han sido los siguientes ${ }^{8}$ :

- Calidad de la Educación: se considera la cantidad de graduados de la institución que han obtenido premios Nobel o medallas Field.

- Calidad de la plana docente: se mide considerando los indicadores, la cantidad de docentes de la institución ganadores de premios Nobel o medallas Field, y la cantidad de docentes cuyos trabajos son citados muy frecuentemente.

- Resultados de investigación: se mide considerando también dos indicadores, los artículos publicados en las revistas Nature y Science, y los articulos citados en el Science Citation Index exoanded y en el Social Sciences Citation Index.

- Dimensión de la institución: se mide dividiendo el puntaje total obtenido en los indicadores anteriores entre el total de docentes a tiempo completo de la institución.

En el ranking de las mejores 500 universidades del mundo obtienen los veinticinco primeros puestos universidades norteamericanas, de las cuales 24 son de USA y una del Canadá, apareciendo entre las cinco mejores universidades las siguientes: Harvard, Stanford, California, Berkeley, Massachusetts Inst Tech y California Inst Tech. En cuanto a la presencia de las universidades latinoamericanas, entre las quinientas, solo figuran siete, cuatro de Brasil, una de México, una de Argentina y una de Chile, destacando por el lugar, la Universidad de Sao Paulo (Brasil en el puesto 153 y la Universidad Autónoma de México en el puesto 201). 
Es importante destacar algunas observaciones señaladas por Carlos García Bedoya ${ }^{9}$ : "Como lo reconocen los propios autores, hay un sesgo muy marcado hacía las ciencias básicas y las ciencias médicas, dándosele escaso peso a las ciencias sociales y prácticamente nulo a las ciencias humanas. Es verdad que en esos campos del saber no se han elaborado indicadores internacionales suficientemente estandarizados, pero se hace necesario afinar instrumentos para evaluar mejor el aporte de estos campos del saber. Los indicadores empleados miden fundamentalmente el desempeño de la institución en el ámbito de la investigación (lo cual es correcto), pero sería importante complementar este aspecto esencial con los indicadores que midan también el desempeño en el ámbito de la enseñanza-aprendizaje. Se privilegia de modo un tanto desmedido el mundo académico anglosajon. Si bien la importancia y calidad de éste resulta indiscutible, no deja de sorprender la posición relativamente rezagada de, por ejemplo, las universidades alemanas.

Sobre el curriculo universitario revisemos algunas conceptualizaciones, citando a Robert Norton del Centro de educación y adiestramiento para el empleo, colegio de educación de la universidad del Estado de Oshio:

El Curriculum es "la descripción o composición de enunciados sobre "lo que debe aprender un estudiante en un programa instruccional especifico; producto que establece los "resultados esperados del aprendizaje ${ }^{10 "}$.

DACUM siglas (Developing a curriculum) "para desarrollar un curriculum". Es un planteamiento del análisis ocupacional que consiste en reunir a un comité de expertos ocupacionales bajo el liderazgo de un facilitador adiestrado. Se utiliza técnicas del "torbellino de ideas" modificar detalladamente los deberes y operaciones que deben realizar los trabajadores para tener éxito en su ocupación ${ }^{11}$.

En cuanto a la Competencia esta es "la descripción de la habilidad que uno posee cuando puede ejecutar una operación ocupacional dada eficiente y eficazmente ${ }^{12 "}$.

La enseñanza basada en competencias (EBC) es un programa instruccional que deriva su contenido de operaciones verificadas (validadas) y basa su evaluación en el desempeño del estudiante. Los materiales educativos utilizados en estos programas identifican, verifican y publican por adelantado las operaciones instruccionales (Competencias) que el estudiante debe aprender y ejecutar, y las condiciones bajo las cuales se llevará a cabo la evaluación. La instrucción resalta la destreza para realizar un trabajo, así como el conocimiento del cómo y el porqué. La ejecución y el conocimiento del estudiante se evaluarán por separado en base a criterios establecidos, en vez de, en base a las normas del grupo ${ }^{13 "}$.

Juan Carlos del Bello en un informe presentado al Banco Mundial en el año $2002^{14}$ sostiene que, "en las últimas décadas del siglo XX se produjeron cambios estructurales en los sistemas educativos en general y en el sistema de educación superior en particular, del cual las universidades forma parte. Sin pretender agotar un listado de estos cambios, se destacan los siguientes: (i) Masificación de la educación superior y creciente inequidad, ii) Crisis del financiamiento público de la educación superior, (iii) Emergencia de la sociedad del conocimiento, iv) Nuevos patrones de la competencia económica internacional, v).Nuevas demandas sociales sobre el sistema universitario, (vi) Desajustes estructurales del mercado de trabajo, vii) Internacionalización de los sistemas de educación superior, (viii) Mayor movilidad del capital humano, (ix) Crisis de la organización estatal del servicio educativo 
universitario, (x) Crisis de los sistemas de gobierno de las universidades, (xi) Redefinición del concepto de autonomía universitaria".

José J. Bruner en un trabajo de investigación sobre el crecimiento de la población universitaria ha establecido que de 600,000 estudiantes que existian en 1960 se pasó a ocho millones en $1995^{15}$.

Estas características latinoamericanas se expresan en el Perú por un crecimiento de universidades privadas, en unos casos por iniciativas particular y en otros el proceso de conversión de Institutos tecnológicos en universidades. Esto tuvo su máxima expresión en la década del 90.

Tanto el concepto de calidad como la evaluación de la calidad universitaria se encuentran rodeados de un conjunto de atribuciones conceptuales parciales o aisladas, siendo oportuno citar documentos de entidades que vienen trabajando sobre el tema en nuestro continente, así tenemos al CNA de Colombia que sostiene que "al estudiar la literatura y observar las experiencia de otros países en materia de evaluación de la calidad, se advierte que, en muchos casos, se utilizan modelos basados en uno de los aspectos que la constituyen. Así, por ejemplo, se juzga que existe calidad en una institución por su reputación, o se asume que la hay cuando ella dispone de los recursos académicos o financieros adecuados, o porque ha obtenido determinados resultados en alguna de sus funciones sustantivas, o por el valor intrínseco de los contenidos académicos de sus programas de formación, o por la apreciación del valor agregado de la educación que imparte, es decir, por lo que el estudiante aprende durante su permanencia en la institución. En otros casos, se identifica la calidad con la mayor o menor satisfacción de estándares fijados por asociaciones profesionales o por agencia de acreditación, o por la satisfacción manifiesta de los empleadores. Con base en tales preferencias, se eligen las estrategias y las metodologías de evaluación de la calidad, condicionando de esta manera la amplitud del concepto mismo de calidad y los alcances de los resultados de su evaluación ${ }^{16 "}$.

De la literatura especializada hemos seleccionado el siguiente concepto de acreditación: "es el reconocimiento público de un programa que cumple determinada calidad profesional y estándares en educación, a través de una evaluación inicial y periódica. Esta relacionado con el aseguramiento de calidad y el mejoramiento de los programas y se aplica a éstos, a diferencia de la certificación o licenciatura que se aplica a los individuos ${ }^{17 "}$.

"La Acreditación trasciende la formalidad de los requerimientos legales para desarrollar y dinamizar un proyecto en el que cada integrante de la comunidad es parte y cuya finalidad ha de responder por una formación integral ética de sus estudiantes así como con calidad académica y carácter humanizador de la persona y de la comunidad; en consecuencia la acreditación debe abordarse como un problema tanto ético como epistemológico que concibe a la institución en su totalidad, como un proceso integrador y válido socialmente, que trasciende la yuxtaposición de procesos, de núcleos pedagógicos, de acciones educativas de sus docentes y de compromisos y acciones de formación adelantadas por sus estudiantes ${ }^{18 "}$.

Pedro Suarez Ruiz (2000) señala las siguientes premisas metodológicas de la acreditación de la calidad ${ }^{19}$ :

1. La acreditación de la calidad educativa avala un proyecto con carácter eminentemente pedagógico enraizado en los demás proyectos de una nación, razón por la cual acredita su calidad pedagógica teniendo esta ciencia como disciplina fúndante. 
2. La acreditación de la calidad educativa es un proyecto con calidad ética no solo porque sea un proceso bueno (Men 2000), sino por ser un proyecto equitativo en tanto justo porque hace posible la vigencia de lo público como espacio que resguarda la dignidad humana y el conocimiento como bien común y por lo tanto al alcance de todos.

3. La acreditación de la calidad educativa avala la equidad no como una oferta de oportunidades iguales para todos, sino como la opción que tiene cada ciudadano y cada región de acuerdo con su singularidad y su contexto para que desde su propio proyecto de vida pueda ser parte estructural de un proyecto comunitario local y nacional.

4. La acreditación de la calidad educativa avala un proyecto que responde a las necesidades de la región y dispone en su estructura u conocimiento legitimado socialmente. Ello significa que no asume solamente el sujeto en su totalidad sino también la comunidad en su totalidad como referente.

En las Memorias del II Congreso Iberoamericano de Psicología y del Primer encuentro iberoamericano de decanos de Psicología (2002) se describe las experiencias de acreditación en México, en los siguientes términos: La acreditación, como un proceso, surgió a partir de una autoevaluación orientada por un grupo de pares, designados por su calidad académica por el comité. Para su desarrollo se han definido perfiles, basados en siete tipo de competencias, tres de las cuales son netamente curriculares y consiste en los siguientes aspectos : conceptual, metodológico (compuesto por lo cualitativo y lo cuantitativo, haciendo énfasis en los aspectos psicometricos) y técnico que se basa en la observación, el seguimiento y las técnicas de diagnóstico. Los cuatro restantes son netamente metacurriculares y son la competencia integrativa, la competencia adaptativa (en la cual se hace énfasis en el "aprendiendo aprender", en donde se busca que el egresado sea autodidacta, además se tenga en cuenta el uso de herramientas tecnológicas). De igual forma, se deba hablar tanto de la competencia ética como contextual.

De otro lado es importante señalar los principios para la formación de psicólogos planteados por el grupo de países pertenecientes al MERCOSUR y en los siguientes términos:

1. Garantizar una formación básica común para el reconocimiento en todos los países de la región:

- Procesos psicológicos cognitivos, motivacionales, de aprendizaje, afectivoemocionales, percepción, atención, interaccionales, del desarrollo, de personalidad, psicopatológicos.

- Historia, teoría y sistemas psicológicos.

- Formación en investigación psicológica.

- Evaluación y diagnóstico psicológico.

- Epistemología de la psicología.

- Psicologías aplicadas (tradicionales y emergentes).

2. Garantizar el pluralismo teórico y metodológico en la formación científico profesional del psicólogo. Programar el acceso a ejes temáticos en el curriculum, desde diversos enfoques teóricos y metodológicos. 
3. Garantizar en el grado de formación generalista y suficiente para el ejercicio profesional, reservando la especialización en el post grado. Prever una carga horaria mínima de 3500 horas cronológicas, la ponderación equilibrada entre teoría, practica e investigación, y la inclusión de un mínimo de 350 horas de practica institucional supervisada. La formación generalista puede incluir orientación de pregrado.

4. Garantizar la formación interdisciplinar Deben incluirse líneas de formación convergente: filosofía, Antropología, sociología, Economía, Matemáticas, Biología, Genética, Lingüística, Estadística, etc. y asignaturas complementarias: Inglés, computación, culturales, etc.

5. Garantizar una formación científica, reconociendo a la psicología como una ciencia que produce conocimientos que aplica Incluir una formación metodológica teórica práctica, atravesando todos los niveles formativos; deben existir requisitos curriculares de presentación de trabajos escritos (ensayos, comunicaciones científicas, informes, tesis, memorias) y formación en competencias lingüísticas.

6. Garantizar la integración teórica-práctica en todo el transcurso del desarrollo curricular. La integración teórica-práctica debe explicitarse en el desarrollo curricular, y debe contar con infraestructura y recursos adecuados (tecnología, laboratorios, gabinetes y otros), debiendo existir una distribución proporcionada de horas teóricas y prácticas con incremento de éstas últimas en los niveles superiores de formación.

7. Garantizar la formación para el trabajo en equipos multiprofesionales: Deberá existir formación en competencias inter e intrapersonales, las que deberán estar explicitadas en el diseño curricular; deberá asimismo promoverse el desarrollo de prácticas inter y transculturales.

8. Garantizar una formación competitiva con la atención a las problemáticas sociales: Deberán definirse objetivos curriculares que posibiliten la formación y la práctica social de la disciplina en instituciones y contextos socioculturales diversos.

9. Promover la construcción de la identidad profesional del psicólogo: Deberán organizarse actividades que propicien contactos con psicólogos en diversas áreas e instituciones. Deberán desarrollarse competencias de actuación, inserción e intervención, adquiridas en practicas supervisadas, y deberán implementarse una política de difusión del quehacer profesional, velando por la presencia y el rol del psicólogo en la sociedad.

10. Promover el compromiso ético a lo largo de la carrera, favoreciendo la formación de actitudes criticas y reflexivas: Deberán desarrollarse competencias ético-axiologicasdeontologicas en todos los cursos de todos los niveles, y se encaminarán a la defensa de los principios de derecho, integridad y dignidad de las personas. Esta formación se dará en interacción con contextos donde aplicar lo aprendido.

Los integrantes del grupo de formación que redactaron el presente documento: Cristina Di Domenico (Argentina), Rocio Peredo (Bolivia), María De Lourdes Contini y Daniela Schhneider (Brasil), Sonia Salas, Adriana Massardo y Carmen Bonnefoy (Chile), Antonia De Millot, Yeny Aguilera de Zarza y Clori de Gomez (Paraguay) y Alicia Cabezas (Uruguay), firmaron el documento: Lic. Mario Molina (Presidente de la Federación de Psicólogos de la República de Argentina), Lic. Rene Calderon (Presidente del Colegio de Psicólogos de Bolivia), Ps. Ana Marcés Bahía Bock (Presidente del Conselho Federal de Psicología de la 
República Federativa de Brasil), Ps. Carlos Urrutia (Presidente del Colegio de Psicólogos de Chile), Lic. Diana S, Lesme R. (Presidenta de la Sociedad Paraguaya de Psicología), Lic. Grisel Añon (Secretaria general Coordinadora de Psicólogos de Uruguay.

Finalmente, tomemos como referencia al espacio europeo, teniendo en cuenta por su puesto, sus condiciones de desarrollo y por tanto las diferencias especificas, ya que en esta región se viene llevando a cabo una experiencia sobre la calidad universitaria. Se inicia por la voluntad política de los gobiernos en la búsqueda de una armonización de la educación superior. Allí tenemos las declaraciones de la Sorbona, Boloña y Praga, realizadas en los años 1998, 1999 y 2001, que apuntan a fortalecer la sociedad del conocimiento y un protagonismo internacional sobre el tema. Revisemos el proyecto Tuning (Tuning Educational Structures in Europe $)^{20}$ compuesto por 100 universidades para alcanzar puntos de referencia, respetando su autonomía y diversidad o riqueza cultural en la construcción curricular, en su proyección de dos fases se han planteado objetivos y líneas de trabajo. Objetivos de la primera fase: (1) Estudiar la convergencia europea en la definición de contenidos y perfiles profesionales en cada área, (2) Facilitar las transparencia entre instituciones definiendo un modelo de "buena practica" basado en objetivos, competencias y buenos resultados, (3) Desarrollar modelos de estructuras curriculares (pre grado y post grado) para cada área para mejorar la integración y el reconocimiento de títulos, (3) Adoptar los créditos europeos de transparencia y acumulación, (4) Revisar parámetros de calidad, (5) Colaborar de forma coordinada con todas las estructuras universitarias (Ministerios, Conferencia de Rectores y Universidades). Estos objetivos activan las siguientes cuatro líneas de trabajo: Línea 1: Competencias académicas generales en todas las titulaciones, Línea 2: Competencias especificas de cada titulación (conocimientos y destrezas), Línea 3: sistema de acumulación, Línea 4: Métodos de enseñanza, aprendizaje y evaluación. Objetivos de la segunda fase: (1) Validación y Consolidación de los resultados de la primera fase: Líneas 1 y 2 (Competencias genéricas y especificas en pre grado y post grado con descriptores de niveles), debate en universidades, empresas, estudiantes, agencias de calidad, etc., Ampliar asociaciones con redes temáticas (2) Nuevas actividades: Ampliar el estudio a nuevas universidades y nuevos países (3) Ampliar el estudio a dos nuevas áreas: Enfermería y estudios europeos (intrerdisciplinar) (3) Objetivos generales: Facilitar las transparencia entre instituciones definiendo un modelo de "buena práctica" basada en objetivos, competencias y resultados y, colaborar en forma coordinada con Universidades, Ministerios, Conferencia de Rectores (EUA) y otras asociaciones y Agencias de calidad.

\section{MÉTODOS}

La complejidad conceptual y la existencia de modelos contradictorios para evaluar la calidad universitaria, configuraron a la presente investigación como exploratoria.

Pues nos encontramos en el camino de búsqueda y construcción de alternativas integrales para realizar investigaciones mayores acerca de la calidad universitaria.

La investigación centralizó su interés en identificar y conocer el trabajo realizado por los organismos rectores de la evaluación de la calidad en los países latinoamericanos, así mismo familiarizarse con las principales experiencias realizadas en nuestro país por tres 
organismos corporativos, en dos de los cuales viene participando activamente la Universidad Nacional Mayor de San Marcos.

Las principales referencias sobre el funcionamiento universitario en la Facultad de Psicología han sido el SUM y los documentos del funcionamiento académico de la Universidad (Plan Estrategico 2002-2006), y de la Escuela Académico Profesional de Psicología. El sistema Unico de Matrícula (SUM) en la UNMSM funciona desde mediados de la década del 90, habiendo creado la agenda Académica Virtual compuesta por lo siguiente ${ }^{21}$ : (1) El Plan de Estudios vigente, (2) La sumilla de cada curso, (3) Los cursos que se encuentran activos en el semestre en curso, sus horarios, secciones y los docentes a cargo del dictado de los cursos, (4) La relación de docentes que se encuentran dictando algún curso en la EAP en el semestre en curso, (5) El currículo resumido de cada uno de los docentes que dictan algún curso para la EAP, (6) El calendario académico del semestre con fecha de matricula, inicio de clases, exámenes parciales, último día de clases, exámenes finales, exámenes sustitutorios, entrega de calificativos y llenado de actas, (7) Procedimientos a seguir por un alumno para matricularse, reserva de matricula, anular matricula, obtener certificado de estudios, obtener grado de bachiller, optar el titulo profesional y otros. La agenda académica debe tener vigencia semestral o anual (según el plan de estudios sea semestral o anual.

\section{RESULTADOS}

En la mayoría de países latinoamericanos existen organismos oficiales que actúan como entes rectores para evaluar la calidad universitaria, así tenemos en Argentina la Comisión Nacional de Evaluación y Acreditación Universitaria (CONEAU), Colombia el Consejo Nacional de Acreditación (CNA), En Brasil desde 1993 la Comisión Nacional de Evaluación de Universidades Brasileñas, y la CONAES (Comisión Nacional de Evaluación de la Educación Superior) en México en 1989 se crea la CONAEVA (Comisión Nacional de Evaluación de la Educación Superior, en el año 2000 se crea el COAPES (Consejo para la acreditación de la Educación Superior), en Costa Rica existe el SINAES (Sistema Nacional de Acreditación de la Educación Superior. En Venezuela el SEA (Sistema de Evaluación y Acreditación de las Universidades Nacionales). En Chile la CNAP (Comisión Nacional de Acreditación de Pre Grado.

El CONEAU (Argentina) es un organismos descentralizado que funciona en jurisdicción del Ministerio de Cultura y Educación, habiendo sido creado en el año 1995 por la Ley de Educación Superior No 24521. La CONEAU está integrada por doce miembros de reconocida jerarquía académica y científica, con experiencia en la gestión universitaria. Ejercen sus funciones a titulo personal, con independencia de criterio y sin asumir la representación de ninguna institución. Su designación se realiza a propuesta de instituciones culturales, universitarias y políticas de la nación argentina.

En el Perú no existe un organismo rector encargado de evaluar la calidad universitaria, habiéndose creado un organismo que mas bien facilita la formación de nuevas universidades denominado el CONAFU (Consejo Nacional para el Funcionamiento Universitario).

Los escasos antecedentes en nuestro país los identificamos de manera corporativa, como la experiencia del CAFME (Comisión para la acreditación de Facultades o Escuelas de 
Medicina), el Consorcio de Universidades privadas y la Alianza Estratégica de Universidades públicas (San Marcos, La Agraria y la UNI).

En América Latina se ha formado el "Mecanismo Experimental de Acreditación (MEXA) creado por el MERCOSUR e integrado por Chile, Argentina, Bolivia y Brasil que avanzan en la acreditación de las carreras universitarias con el objetivo que los títulos profesionales tengan validez en estos países.

Sobre el concepto de calidad universitaria, encontramos que involucra temas de pertinencia y equidad, referidos principalmente a su naturaleza histórica y cultural, de ser promotora del conocimiento y desarrollar una comunidad académica sensible con las desigualdades sociales existentes en la época. No se puede pues conciliar, bajo el riesgo de no hacer un autentico análisis de la calidad, al someter a las universidad a la lógica del mercado, la competencia y el liderazgo empresarial, aunque podemos responder que la universidad no esta aislada de esas dimensiones, pero tampoco esta subordinada a ellas. La universidad debe ser promotora del compromiso social produciendo conocimientos y formando ciudadanos profesionales que busquen las soluciones más adecuadas para el progreso de la sociedad, pese a que reconozcamos Delors que la globalización trae consigo la "desilusión del progreso ${ }^{22}$ ".

El Plan Estratégico Institucional (PEI) 2002-2006 de la Universidad Nacional Mayor de San Marcos, en los lineamientos de política establece tres aspectos fundamentales: a. Excelencia académica, 2. Modernización Administrativa y 3. Cultura Organizacional. Desagregando cada uno de ellos textualmente se indica lo siguiente, sobre la excelencia académica" Sustentada en una eficaz y eficiente organización académica, con currículos actualizados y potencial humano altamente calificados, acreditados internacionalmente, que garantice la formación de profesionales cultos, generadores de conocimientos que contribuyan con el desarrollo del pais y del mundo ${ }^{23 "}$, sobre la modernización administrativa "Optima gestión en todas las instancias de la universidad, con eficiencia y simplificación administrativa, propiciando la integración institucional al servicio de la excelencia académica $^{24 "}$, sobre la cultura organizacional "Una comunidad universitaria institucionalizada, legitimada a través de sus órganos de gobierno, basada en principios y valores hacía una gestión de alta calidad ${ }^{25}$ ".

En el año 2001 se crea la Oficina Técnica del Estudiante, mediante RR. N ${ }^{\circ}$ 06221-R-01, el 13 de setiembre del 2001. "Con ello se trata de conocer la realidad, las necesidades, y al mismo tiempo los problemas que tienen los estudiantes a nivel personal así como en las diferentes áreas de su vida. A partir de ello se busca implementar medidas académicas y administrativas que mejoren el funcionamiento institucional, así como crear políticas sociales y culturales que generen altos niveles de bienestar entre el estudiantado ${ }^{26 "}$.

La formación profesional del Psicólogo en San Marcos esta organizada en las siguientes áreas: (1) Area propedeutica y de los procesos psicológicos básicos, (2) Área Técnico Instrumental, (3) Área Clínica, (4) Área Educativa, (5) Ârea Social Laboral y (6) Área de Prácticas preprofesionales de internado en Psicología.

Sobre la enseñanza de psicología, encontramos que en el año 2001 se modifico el silabo produciendo un documento técnico orientador ${ }^{27}$, en cuya presentación se especificaba lo siguiente "la necesidad de modificar sustancialmente la educación universitaria se ha convertido en un tema de debate permanente entre docentes y los alumnos como protagonistas, más aún cuando nos encontramos en un periodo a recobrar el liderazgo académico y profesional 
de San Marcos que siempre la caracterizó, fundamentalmente por aportar los modelos alternativos que fielmente fueron asumidos por las otras universidades. Un segundo criterio, es el inicio de un nuevo milenio que se presenta con una carga abundante de información tipificada como hiperinformación, exigiendo una mejor predisposición para planificar la actuación de la enseñanza universitaria, como un aspecto especializado y como criterio para medir la calidad de la enseñanza".

En efecto, se introdujo un nuevo modelo de silabo, estableciendo las normas generales y las normas académicas.

En cuanto a datos empíricos en la Facultad de Psicología, hemos tomado el prediagnostico realizado por el gobierno transitorio $(2000,2001)$, donde se estableció que, desde el año de ingreso hasta la obtención del bachillerato académico, el $62 \%$ de los estudiantes se demoraban entre 6 a 10 años, de 11 a 15 años el $25 \%$, de 16 a 20 años el $9 \%$ y de 21 años y más el $4 \%$.

Sobre el tiempo transcurrido desde la obtención del bachillerato a la obtención del titulo profesional de Psicólogo: El mismo año que obtuvo el grado de Bachiller, el 22\%, un año después el $39 \%$, dos años después, el $12 \%$, tres años después el $10 \%$, cuatro años después el $6 \%$, cinco años después el $4 \%$, seis años después $3 \%, 7$ años después 2 , un año después $1 \%$, ocho años después $1 \%$.

Sobre la graduación en Post Grado, al concluir el año 2000, se identifican los siguientes indicadores: el año 2000: 05 magíster, el año 1999: dos magíster, el año 1998: siete magíster, el año 1997: dos magíster.

\section{DISCUSIÓN}

La Universidad Nacional Mayor de San Marcos en el año 2003 presentó a la comunidad Universitaria los "lineamientos para una política de calidad, autoevaluación y acreditación" elaborado por los docentes Zenón Depaz Toledo y Juana Cuba Sancho. Este primer documento presenta una matriz compuesto por dos ejes y nueve factores, que orientan la autoevaluación en nuestra universidad

Reconociendo este documento como pionero en su genero, y teniendo la tarea de adecuar la matriz de autoevaluación, presentamos una alternativa especifica para la autoevaluación de la cerrera profesional de psicología, para aplicarse en la UNMSM y/o en otras universidades que forme psicólogos profesionales, teniendo como tarea inmediata la definición de estándares mínimos de calidad.

1. Proyecto de desarrollo universitario

1.1. Paradigma de universidad

1.2. Visión y misión universitaria

1.3. Presencia en la realidad nacional

1.4. Logros obtenidos institucionalmente

1.5. Integración de red universitaria

1.6. Organización de eventos académicos (nacionales e internacionales) 
2. Gobierno de la facultad y gestión

2.1. Consejo de facultad

2.2. Composición docente y estudiantil

2.3. Normas para la toma de decisiones

3. Producción de conocimientos

3.1. Líneas de investigación

3.2. Funcionamiento del Instituto de Investigaciones Psicológicas

3.3. Docentes investigadores

3.4. Publicación de investigaciones

4. Normatividad de funcionamiento de la Facultad de Psicología y/o Escuela Académico Profesional

4.1. Acta de fundación y/o resolución de creación

4.2. Estructura académica y administrativa

4.3. Reglamentos y manual de procedimientos

5. Perfiles docentes

5.1. Cuadros académicos docentes (grados y títulos)

5.2. Condición laboral (estables, nombrados y contratados)

5.3. Categoría y clase docente

5.4. Organización académica docente (secciones)

5.5. Producción intelectual

5.6. Participación anual en eventos académicos

6. Perfiles estudiantiles

6.1. Criterios de admisión universitaria

6.2. Criterios de permanencia

6.3. Perfiles de competencias: de ingreso, de proceso y de egreso

6.4. Política de estímulo a los estudiantes

6.5. Tiempo de permanencia para concluir sus estudios

7. Propuesta curricular

7.1. Fundamentación académica (paradigmas y áreas de conocimiento)

7.2. Fundamentación profesional del psicólogo

7.3. Sistema de enseñanza

7.4. Duración de la carrera 
Revista de Investigación en Psicología - Vol. 8, N. ${ }^{\circ} 2$

7.5. Áreas curriculares

7.6. Clasificación de las asignaturas

7.7. Plan de estudios

7.8. Prácticas preprofesionales

7.9. Vigencia del actual currículo

8. Servicios de Bienestar y Tutoría

8.1. Programas de Bienestar

8.2. Programas de Orientación al estudiante

8.3. Sistema de Tutoría

9. Equipamiento técnico Pedagógico

9.1. Infraestructura Pedagógica: Aulas

9.2. Laboratorios de Psicología Experimental

9.3. Gabinetes de Tests Psicológicos

10. Recursos bibliográficos

10.1. Funcionamiento de la biblioteca especializada

10.2. Ingreso anual de títulos bibliográficos

10.3. Ingreso anual de Revistas especializadas

10.4. Consulta bibliográfica: Presencial y virtual

11. Soporte de nuevas tecnologías informáticas y Comunicación

11.1. Funcionamiento de la Oficina de Informática

11.2. Informatización de los procesos de matrícula

11.3. Informatización de actas de calificación de asignaturas

11.4. Informatización de la consulta bibliográfica

11.5. Funcionamiento de la enseñanza virtual

11.6. Equipamiento para Teleconferencias

12. Grados y Títulos

12.1. Requisitos para optar el Grado de Bachiller

12.2. Requisitos para optar el Título de Psicólogo

12.3. Requisitos para optar el Grado Académico de Magíster

12.4. Requisitos para optar el Grado académico de Doctor

12.5. Requisitos para los estudios de Diplomatura 


\section{Egresados}

13.1. Registro de egresados

13.2. Protagonismo en los campos especializados

13.3. Protagonismo en la política nacional

13.4. Mercado laboral (perfil ocupacional)

14. Red orgánica Universitaria a la que pertenece

14.1. Comunicación con universidades extranjeras

14.2. Comunicación con universidades nacionales

14.3. Sistema de elección del par externo

15. Apoyo administrativo

15.1. Funcionamiento Administrativo

15.2. Manual de procedimientos Administrativos

16. Infraestructura

16.1. Edificación especializada

16.2. Aulas y equipamiento

16.3. Laboratorios y equipamiento

16.4. Biblioteca especializada

17. Extensión Universitaria y Proyección Social

17.1. Promoción del Psicólogo Profesional : Publicaciones y Eventos

17.2. Promoción de la Psicología como conocimiento especializado : Eventos

17.3. Centros comunales de docencia y entrenamiento profesional

17.4. Cursos permanentes de extensión para la difusión de la Psicología: Soportes académicos y especializado a profesionales afines

18. Presupuesto

18.1. Presupuesto anual

18.2. Inversión por estudiante

18.3. Ingresos brutos

18.4. Egresos

19. Proyectos innovadores

19.1. Planes de mejora (ejecutados y/o proyectados)

20. Proyecciones para el futuro

20.1. Para el desarrollo de la formación profesional

20.2. Para el desarrollo de la investigación 
20.3. Para el desarrollo docente

20.4. Para el desarrollo de la extensión universitaria

\section{CONCLUSIONES}

1. En el Perú se ha difundido modelos teóricos de calidad, importados de las certificaciones de calidad de las empresas productivas y de servicios.

2. Las tendencias conceptuales internacionales sobre calidad universitaria inciden en los indicadores de investigación y/o producción de conocimientos y el impacto de los egresados en la cultura universal.

3. Existen dos referencias internacionales para reorientar la formación de Psicólogos en el Perú: El proyecto Tuning y los principios de formación de psicólogos de MERCOSUR.

4. La Universidad Nacional Mayor de San Marcos tiene una matriz para realizar la autoevaluación que conduzca a la acreditación, debiendo de adecuarla a cada una de las Facultades.

5. Se propone un modelo alternativa para realizar la evaluación de la formación profesional de psicólogos, quedando como tarea de investigación establecer los estandares mínimos de funcionamiento para lograr la calidad.

\section{REFERENCIAS BIBLIOGRÁFICAS}

1. Abad, Diario y otros (2003). Lineamientos para la acreditación de programas. Bogotá: CNA.

2. Aljovín, C. y Germaná C. (2002). La universidad en el Perú. Lima: Fondo Editorial UNMSM.

3. Alvarez , V. y Lázaro, A. (2002). Calidad de las universidades y orientación universitaria. Málaga: ALJIBE Ediciones.

4. Burga, Manuel (2005). Un nuevo San Marcos para el Perú del siglo XXI. Lima: Rectorado UNMSM.

5. Compromiso con la Innovación y el cambio (2004). Principios, lineamientos y programa. Lima: Folleto.

6. Depaz, Z. y Cuba J. (2003). Lineamientos para una política de calidad, autoevaluación y acreditación. Lima: OCAA UNMSM.

7. EAP de Psicología (2001) Lineamientos sobre la elaboración y ejecución del sílabo en la EAP de Psicología Lima: UNMSM.

8. Guzmán Vargas, Oliveira, J. y Paredes, Gerson (2002). Diálogos institucionales: Lima: Fondo Editorial UNMSM.

9. Norton, Robert (2002) Seminario Internacional: Competencias profesionales. Visiones de la Universidad y la Empresa. Lima: UPC.

10. OCCAA (2005) Hacia la autoevaluación en San Marcos Lima: OCCAA UNMSM. 
11. Orellana, Oswaldo (2003). «Reforma curricular en psicología». En Revista de Psicología. Año VI, Nº 1, Lima. Fac. de Psicología UNMSM.

12. OTE (2001). Encuesta de opinión y Expectativas: El estudiante sanmarquino 2001. UNMSM: OTE.

13. Suárez, Pedro (2000). ¿Cómo acreditar su institución? Fundamentos y metodología Bogotá: Orion Editores.

14. Toro, J. y Villegas, Julio (2001). Problemas centrales para la formación académica y el entrenamiento profesional del psicólogo en las Américas. Argentina: SIP.

15. UNMSM (2004) Ranking Universitario Mundial: Elaborado por la Universidad de Shanghai-China Lima: UNMSM.

Notas

1 Chomsky N. y Dieterich, H. (1997). La aldea global F Nafaroa: TXalaparta, p. 87.

2 Ortega y Gasset, J. (1997). Misión de la Universidad, Madrid: Alianza.

3 Hebe M.C. Vessuri (1993). Desafíos de la educación superior en relación con la Oformación y la investigación ante los procesos económicos actuales y los nuevos desarrollos tecnológic en la Revista Iberoamericana de Educación Número 2: Educación, Trabajo y Emple (mayo-agosto, 1993).

4 Acuerdo Institucional Sanmarquino (2004) Balance y propuesta Lima: AIS.

5 Idem pp. 5-6.

6 UNMSM (2002) Plan Estratégico Institucional (PEI) 2002-2006, p. VI.

7 Hebe M.C. Vessuri (1993). Desafíos de la educación superior en relación con la Oformación y la investigación ante los procesos económicos actuales y los nuevos desarrollos tecnológic en la Revista Iberoamericana de Educación Número 2: Educación, Trabajo y Empleo (mayo-agosto, 1993).

8 UNMSM (2004). Ranking Universitario mundial elaborado por la Universidad de Shanghai-China, p. 7

9 IDEM, p. 8.

${ }^{10}$ Norton, Rober (1991) Manuaol SCID, p. 6.

11 IDEM, p. 6

12 IDEM, p. 6

13 IDEM p. 6.

${ }^{14}$ Del Bello, Juan Carlos (2002) Desafios de la política de educación superior en América Latina: Reflexiones a partir del caso argentino con énfasis sobre la evaluación para el mejoramiento de la calidad Bogotá: The World Bank, pp. 1-2.

${ }_{15}$ Brunner R. José J. (2000) Educación superior y desarrollo en el nuevo contexto latinoamericano: Los sistemas de educación Chile: Revista de Educación Superior.

${ }^{16}$ CNA(2002) Lineamientos para la acreditación Colombia: CNA.

${ }^{17}$ Acreditation manual (2000) $9^{\text {th }}$ edition American.

${ }^{18}$ Suárez Ruiz, Pedro (2000) ¿Cómo acreditar su instituciómn? Fundamentos y metodología, p. 8.

${ }_{19}$ IMEM, p. 8.

20 http:www.relint.deusto.estuningprojet/index.htm

21 Informativo del SUM (2003) Editorial Año 2, № 7, Lima: SUM UNMSM.

22 Delors, Jacques (1998) La educación encierra un tesoro. Bogotá: UNESCO.

${ }^{23}$ UNMSM (2002)Plan Estratégico Institucional (PEI) 2002-2006, p. IV.

${ }^{24}$ IDEM, p. IV.

${ }^{25}$ IDEM, p. IV.

${ }^{26}$ OTE (2001) Encuesta de opinión y expectativas: El estudiante sanmarquino 2001, p. 1.

${ }^{27}$ EAP de Psicología (2001). Lineamientos sobre la elaboracióny ejecución del silabo en la EAP de Psicología Lima: EAP de Psicología de la UNMSM.

${ }^{28}$ Depaz, Zenon y Cuba, Juana (2003). Lineamientos para una política de calidad, autoevaluación y acreditación Lima, UNMSM. 